\title{
HUMEAN LAWS AND EXPLANATION
}

\author{
BARRY LOEWER \\ Rutgers University, USA \\ loewer@philosophy.rutgers .edu
}

\begin{abstract}
My primary focus in this paper is on an objection to Humean account of laws and specifically to David Lewis' "best systems analysis" (BSA). The objection is that the laws according to the BSA (which I call L-laws) fail to account for the ability of laws to explain. In contrast governing laws (which I will call G-laws) are alleged to account for the role of laws in scientific explanations by virtue of their governing role. If governing is required for laws to be explanatory then Humean accounts like Lewis' are dead in the water since explanation is central to the role of laws in the sciences. However, I will argue that there are effective rebuttals to arguments that Humean laws don't explain and that actually it is governing accounts that have difficulty with explanation.
\end{abstract}

Keywords: Laws of nature $\bullet$ Humean supervenience $\bullet$ best systems analysis $\bullet$ explanation

I have come to think that the laws of physics are real because my experience with the laws of physics does not seem to me to be very different in any fundamental way from my experience with rocks. For those who have not lived with the laws of physics, I can offer the obvious argument that the laws of physics as we know them work, and there is no other known way of looking at nature that works in anything like the same sense.

Steven Weinberg NYR 1996

The idea that physics should aim to find mathematical laws that govern the universe was born in and nurtured by the theology of the $17^{\text {th }}$ century. During that time physicists and philosophers rejected the Aristotelian conception of science with its diversity of systems and explanations in terms of powers, capacities, and goals for a conception of explanation in terms of unified mathematical laws of nature. Descartes, Galileo, Newton and their contemporaries thought of laws as mathematical principles that 
describe how God governs the motions of passive matter. ${ }^{1}$ In Newton's view his mechanical laws explain the motions of the planets because that is how God wills them to move. Thinkers in the $17^{\text {th }}$ century also thought that laws exhibit features that reflect God's nature. Because they are derived from God's nature laws were thought to be unifying, eternal, simple, and exceptionless. ${ }^{2}$ During the following centuries physicists were enormously successful in proposing candidates for laws that exhibit these divinely inspired features. So much so that by the end of the $20^{\text {th }}$ century some physicists dreamed of finding the "final theory" that contained the complete set of laws of the universe (e.g. Weinberg 1993).

But also by the end of the $20^{\text {th }}$ century physicists and philosophers had mostly forgotten the theological origin of the concept of laws. ${ }^{3}$ Nevertheless, both the view that laws govern and the view that they are simple, mathematical components of $\mathrm{A}$ unified and comprehensive system persist in how many physicists and philosophers think and talk about laws today. This leads to these two questions: 1. "Without God how can we make sense of laws governing?" 2. "Why should laws exhibit the previously mentioned divinely inspired features?" And if laws are not principles that describe how God governs nature what makes an equation express a law? Or as Stephen Hawking asks, "What breathes fire into the equations?"

The contemporary philosophical discussion of the metaphysics of laws is dominated by two approaches to answering Hawking's question. I will call them Governing and Humean accounts of laws. ${ }^{4}$ The first takes the idea that laws govern seriously. It claims that it is because laws govern that they possess the power to explain the regularities manifested in our world. While talk of governing echoes the theological birth of the concept of law few of its philosophical defenders make an overt appeal to theology to explicate it. ${ }^{5}$ Rather, they understand laws to be features of reality over and above occurrent events that in some way necessitate or constrain lawful regularities. In contrast, Humean accounts consider governing to be a misleading metaphor left over from the concept of law's theological origin. They construe laws to be scientifically significant truths that systematize phenomena and hold that that are able to play their role in scientific explanations without any reliance on governing. ${ }^{6}$

My primary focus in this paper is on an objection to Humean account of laws and specifically to David Lewis' "best systems analysis" (BSA). The objection is that the laws according to the BSA (which I call L-laws) fail to account for the ability of laws to explain. In contrast governing laws (which I will call G-laws) are alleged to account for the role of laws in scientific explanations by virtue of their governing role. If governing is required for laws to be explanatory then Humean accounts like Lewis' are dead in the water since explanation is central to the role of laws in the sciences. However, I will argue that there are effective rebuttals to arguments that Humean laws don't explain and that actually it is governing accounts that have difficulty with explanation. 
Lewis develops his account of laws within a Humean account of fundamental ontology. He proposes that the actual world consists entirely of the instantiations of fundamental properties at points (or by point size individuals) throughout space-time and that the only fundamental relations are metrical relations. As he says, reality at its most fundamental level consists of "one damn thing after another". There are no G-laws or other items that make for fundamental necessary connections. The totality of fundamental property instantiations throughout space-time is the Humean Mosaic (HM). Lewis calls the thesis that all else supervenes on the HM, "Humean Supervenience". ${ }^{7}$

Hume is usually interpreted as denying the reality of causation (and the other nomological modalities) but this is not Lewis' view. Lewis thinks that causation, laws, counterfactuals, chances are real but that they are not fundamental. Like everything else they supervene on the HM. Lewis argued for this by showing how laws and chances can be characterized together in terms of patterns exhibited by the HM, counterfactuals can be characterized in terms of laws and world similarity, and causation characterized in terms of counterfactuals. If his program is successful it would provide a powerful defense of Humean Supervenience. ${ }^{8}$

According to Lewis, laws are propositions (typically expressed by generalizations or equations) entailed by the scientifically optimal systematization of the HM. He calls this the "Best System Analysis" (BSA) of laws and characterizes it as follows:

Take all deductive systems whose theorems are true. Some are simpler better systematized than others. Some are stronger, more informative than others. These virtues compete: An uninformative system can be very simple; an unsystematized compendium of miscellaneous information can be very informative. The best system is the one that strikes as good a balance as truth will allow between simplicity and strength. How good a balance that is will depend on how kind nature is. A regularity is a law iff it is a theorem of the best system. (1994, p.478). ${ }^{9}$

Objective probabilities enter the picture with laws that specify probabilities. Probabilistic laws earn their place in a best system by increasing informativeness at little cost in simplicity. ${ }^{10}$

A generalization or equation (or the proposition it expresses) is an L-law in virtue of its place in the scientifically best systematization of the Humean mosaic. This is Lewis' answer to Hawking's question. In contrast a G-law isn't itself a generalization but something that makes a generalization true in virtue of governing its instances. This is the governing answer to Hawking's question. So, the governing account promotes one part of the $17^{\text {th }}$ century concept of law - laws govern - while Lewis' Humean account promotes the other part - that laws are specified by a simple, comprehensive system. 
Because of the way that the concept of law is involved in accounts of physical possibility, causation, chance, counterfactuals, scientific explanation and so on there are governing and Humean versions of each of these characterized respectively in terms of G-laws and L-laws. On G-accounts counterfactuals are supported by and causal relations are covered by G-laws while on L-accounts counterfactuals are supported by and causal relations are covered by L laws. From the perspective of a proponent of governing accounts each of the Humean versions are incapable of performing the work required of them by science. Humeans should agree that from that perspective these accounts are revisionary but argue that contrary to anti-Humean claims they can play the role they play in science as well or better than governing versions.

Lewis' BSA has many virtues. Chief among these are that it dispenses with the obscure metaphor of governing. ${ }^{11}$ Further, its characterization of laws is connected to scientific practice since criteria for a best systematization - informativeness and simplicity - are valued by physicists when evaluating proposals for fundamental theories. Also it can be naturally extended to accommodate probabilistic laws including probabilities compatible with deterministic dynamical laws (e.g. the probabilities posited in statistical mechanics and Bohmian quantum mechanics). ${ }^{12}$ Despite these virtues the objection is that L-laws fail to be genuine laws since they can't play the role laws play in scientific explanations. ${ }^{13}$

I will discuss two problems regarding explanation that allegedly face L-laws. The first is that L-laws do not explain in the way that laws explain in ordinary scientific explanations. The second is that if the world were Humean (i.e. it lacked G-laws) the existence of lawful patterns would be an astonishing coincidence and for this reason we should reject Humeanism. As we will see, these objections are closely related.

Here is Tim Maudlin arguing that L-laws do not explain:

If the laws are nothing but generic features of the Humean Mosaic, then there is a sense in which one cannot appeal to those very laws to explain the particular features of the Mosaic itself: the laws are what they are in virtue of the Mosaic rather than vice versa. (Maudlin 2007, p.172).

Maudlin's objection is that since the fact that a generalization is an L-law is explained by patterns in the Humean mosaic the L-law can't turn around and explain aspects of these patterns on pain of circularity. There is no such circularity in explanations by G-laws since G-laws are not themselves explained by patterns in the HM but govern them. Since it is one of the jobs of laws to support explanations this objection threatens to be devastating to the BSA and Humean accounts generally.

An initial Humean response to the objection is to distinguish between scientific and metaphysical explanations (Loewer 2012). Without giving a complete account of these two kinds of explanations it is enough for the purposes of this reply to say that the two kinds of explanation are quite different. The relevant kind of scientific 
explanations work by adverting to a law that connects an event to its (or one of its) causes or by subsuming a generalization under a more fundamental generalization. The relevant kind of metaphysical explanations work by showing how a fact obtains in virtue of or is grounded in a more fundamental fact. Scientific explanations of particular events are typically diachronic while metaphysical explanations are typically synchronic. For example, that the balloon burst because the air in it was heated is a scientific explanation. The fact the temperature of a gas is determined by or grounded in the average kinetic energy of its constituent molecules is a metaphysical explanation.

The response to the charge that explanation by L-laws is circular is defused by the observation that an L-law is involved in the scientific explanation of one event in the HM by another while the HM is involved in a metaphysical explanations of why a generalization is an L-law. The circularity is thus removed. ${ }^{14}$

Marc Lange responded to this attempt to defuse the circularity worry by arguing that circularity is restored by applying a transitivity principle that connects scientific and metaphysical explanations. The principle is

(Trans) If E scientifically explains [or helps to scientifically explain] $\mathrm{F}$ and D grounds [or helps to ground] E, then D scientifically explains [or helps to scientifically explain] F. (Lange 2013, p.256).

Lange takes grounding to be a kind of metaphysical explanation. So, if a law L scientifically explains an aspect of the mosaic F since on Lewis' BSA F partly grounds $\mathrm{L}$ it follows from Trans that $\mathrm{F}$ is part of a scientific explanation of itself. Circularity regained! In contrast Trans causes no difficulty for G-laws since they are not grounded in their instances.

Lange supports Trans by providing instances where it seems to be correct. For example, the increase in the temperature of air in a balloon scientifically explains why it burst. ${ }^{15}$ The change in momentum of each of the particles of air in the balloon metaphysically explains the increase in temperature. By Trans the increase in momentum of air particles in the balloon also explains its bursting. That seems right. In examples like this, E scientifically explains $\mathrm{F}$ by being involved in causing $\mathrm{F}$ and so whatever metaphysically determines F must also be involved in causing and thus scientifically explaining F. Trans works for scientific explanations that are causal explanations. It is not surprising that an anti-Humean like Lange thinks that Trans applies to explanation by laws since they tend to think of governing as akin to causing. But Humeans do not think of the explanatory role of an L-law in this way. The role of Humean laws is to describe and systematize not to govern. So, from the Humean point of view Lange's application of Trans to scientific explanation in terms of laws already presupposes the governing account. In view of this diagnosis Humeans should just reject that Trans applies to the way they understand how laws scientifically explain. ${ }^{16}$ 
How then should the role of laws in scientific explanations be understood? The most important explanations involving laws are D-N explanations and causal explanations. In a D-N explanation, a law is an essential component of the explanans that entails the explanandum. There are DN-explanations both of laws in terms of more general laws e.g. the explanation of Kepler's laws in terms of Newton's laws of mechanics and gravitation and of particular events, e.g. explanation of the detection of gravity waves in 2015 in terms of colliding black holes and laws of GR. ${ }^{17}$ The role of L-laws in causal explanations is a bit more complicated. On Lewis' counterfactual account of causation "the heating of the balloon caused it to burst" is true if the counterfactual "if the balloon had not been heated it would not have burst" is true. The law "heating of a balloon under such and such conditions causes it to burst" is involved in the truth conditions of this counterfactual via its role in the similarity relation that explains counterfactuals which in turn account for causation. So, the role an L-law plays in a causal explanation is by being part of the metaphysical explanation of the causal relation between the explanans event and the explanandum event. Advocates of G-laws may object that L-laws don't support counterfactuals or ground causation. But this objection on its own is clearly begging the question.

Of course, the relevant notions of causation, counterfactuals, and explanation are the L versions since they all involve L-laws. L-laws don't explain events by producing or governing them, but they can play the role laws play in scientific explanations of events even if these very events are part of the metaphysical explanation of L-laws. This circularity is unproblematic. As far as accounts of counterfactuals and causation are concerned L-laws are as good as G-laws.

In a recent paper Nina Emery develops a slightly different circularity objection (Emery 2019). She argues that the distinction between scientific and metaphysical explanation fails to defuse the circularity problem for the Humean view since laws metaphysically explain their instances. If, as Humeans maintain, the HM, which includes instances of laws, metaphysically explain laws then there is a circularity of metaphysical explanation. For Emery a law explains its instances if they hold in virtue of the law holding. But why think that laws metaphysically explain their instances? Emery has two arguments. The first is that scientists say that laws explain their instances and that the only way to understand this explanatory relation is either as causation or as grounding. She reasonably says that laws don't literally cause their instances so concludes that they ground their instances. ${ }^{18}$ Grounding is a kind of metaphysical explanation. If $A$ grounds $B$, then $A$ makes $B$ obtain. The idea that laws ground their instances is a suggestion that proponents of G-laws might welcome since to the extent that grounding is understood it provides an account of what it is for a law to govern its instances. But as grounding is usually understood I don't think that laws ground their instances either. I will return to this point later.

Her second argument is that if laws didn't explain their instances then that would 
leave the patterns corresponding to those laws unexplained, and it is a key component of scientific practice not to leave such patterns unexplained. As Emery anticipates, Humeans will reject the premise that Laws explain their instances in the sense required for her argument. Unlike a G-law, an L-law is not an element of reality that makes its instances obtain either by causing or grounding them. It is true that scientists sometimes say that a law explains its instances and mean by this that the law makes the instances obtain. But for a Humean thinking of laws like this is a remnant of the theological origin of the concept of law. A less metaphysically loaded account of explaining a regularity is provided by the D-N account of explaining a lawful pattern in terms of an L-law. Of course, this will leave the most fundamental L-laws unexplained since they cannot be deduced from more fundamental laws. This is the second problem that Humean laws are said to have with explanation. I will address it shortly.

Rejecting the claim that a law explains its instances by in some sense making them obtain is not, as Emery says, scientifically revisionary. But to the extent that one thinks of laws as governing it is metaphysically revisionary since it rejects the governing conception of laws. This is exactly what the Humean proposes.

I want to turn now to a second alleged explanatory deficiency of Humeanism. The charge is that on Humean accounts there is no explanation of why the HM exhibits any lawful patterns in the first place. John Foster lodges the complaint in these passages:

The past consistency of gravitational behavior calls for some explanation. For given the infinite variety of ways in which bodies might have behaved nongravitationally and, more importantly, the innumerable occasions on which some form of non-gravitational behavior might have occurred and been detected, the consistency would be an astonishing coincidence if it were merely accidental - so astonishing as to make the accident-hypothesis quite literally incredible.

What is so surprising about the situation envisaged — the situation in which things have been gravitationally regular for no reason - is that there is a certain select group of types, such that (i) these types collectively make up only a tiny portion of the range of possibilities, so that there is only a very low prior epistemic probability of things conforming to one of these types when outcomes are left to chance. (Foster 2004, p.68)

Foster's claim is that without an explanation, Newton's gravitational regularity would be "an astonishing coincidence". More generally he thinks that any apparently lawful regularity that lacks a scientific explanation is an astonishing coincidence. Humeans can agree that he is correct for non-fundamental regularities. So, for example the Keplerian regularities would appear to be coincidental if they were not explainable in terms of Newton's laws. But the Humean account is committed to 
there being fundamental regularities that have no further scientific explanation. On Lewis' account these are the axioms of the theory that is the scientifically best systematization of the HM. On the Lewisian account there is a metaphysical explanation of why a system best systematizes the HM in terms of the criteria for a scientific systematization and the HM that is systematized but this doesn't explain why the actual HM is systematizable at all. So, the objection is that if the world were Humean then we should not expect it to be systematizable and lawful. It is an astonishing coincidence that it is.

This objection is related to but different from the earlier objection that Humean laws can't explain their instances. Foster's problem is that fundamental L-laws are themselves in need of explanation and even if non fundamental laws can be explained in terms of more fundamental laws the regularities expressed by the most fundamental L-laws have no explanation. This is supposed to contrast with the view that fundamental regularities are expressed by G-laws since they are explained by the G-laws. Of course, for the Humean there is a metaphysical explanation for why a certain theory best systematizes the HM in terms of the entire HM. But to the objector this just emphasizes the fact that the fact that the HM exhibits these patterns is a vast and astonishing coincidence. I will call this "the Coincidence Argument".

Here is an attempt to spell out the Coincidence Argument. ${ }^{19}$ Begin by noting that the set of HMs that are systematizable by a Lewisian best theory are a tiny minority among all the HMs. Lacking any further information, it may seem a priori rational to assign equal epistemic probability to each possible HM. It follows that given Humeanism the probability of the actual HM being one that has a Lewisian best theory is itself very small. In contrast, worlds whose lawful regularities are explained by G-laws are a priori much more probable than worlds whose regularities are coincidences. Since our world apparently has lawful regularities it follows that it is likely that there are G laws; i.e. Humeanism is false. Understanding why this argument fails will strengthen the case for Humeanism.

The crucial premise in the argument is that a world exhibiting a regularity without an explanation has a smaller epistemic probability than a world exhibiting the same regularity which has also has an explanation. Foster motivates this principle with the example of a sequence of coin flips that whose outcomes are all heads. He points out that if this sequence is the result of chance it is a coincidence and far less likely than if it can be explained by a mechanism that causes the coin to land heads (e.g. that the coin is magnetized in such a way that when flipped in a magnetic field it almost always lands heads). This is certainly true, but this reasoning doesn't apply to the case of the axioms of the theory that best systematizes the HM. The instances of these axioms are not the result of chance and according to Humeanism there can be no more fundamental explanation. So, they are not coincidences like a sequence of coin flips that results only in heads. 
A different approach is to understand the probability in the Coincidence argument as rationally required in virtue of the principle of indifference POI. The POI says that given a set of possibilities if one is ignorant of which is actual so that there is no reason to support one over another one should assign equal epistemic probability to each. Since systematizable worlds seem to be a tiny minority of all worlds POI seems to recommend assigning a tiny probability to the actual world being systematizable. ${ }^{20}$

The trouble with his suggestion is that the POI is not itself rational. It is well known that without qualifications it leads to contradictions. But the main problem is that ignorance concerning which world is actual should not be parlayed into a rationally required probability distribution. As far as I can see it is not irrational to assign equal epistemic probability to each HM (relative to a measure) but also not irrational to assign higher epistemic probability to worlds whose HMs can be systematized than to worlds whose HMs have no best system. And there is nothing irrational about assigning 0 epistemic probability to worlds that contain no G-laws or assigning no probability distribution at all.

Does the metaphysical view that laws are G-laws alleviate the feeling of astonishment that the world is systematizable? I don't see that it does. There are uncountably many worlds some of which are Humean mosaics without G-laws and some which are Humean mosaics with G-laws. Those with G-laws are constrained so that they don't violate the regularities associated with their G-laws. But it is just as astonishing as it is for that the actual world is one of those with G-laws that are systematizable. Still one might argue that a view that leaves the regularities expressed by fundamental L-laws without a deeper explanation is unsatisfactory compared to a view on which they are explained. For Descartes laws explain since they are the principles that describe how God directs the motions of bodies. But without divine backing it is unclear how G-laws do their governing and explaining. I will conclude by casting some doubt on the claim that G-laws explain at all. If they don't then it may be irrational to believe that there are G-laws.

Proponents of G-laws often talk as though laws constrain or produce or cause their associated regularities. But it is hard to take this seriously. That would treat a law as some kind of event or condition that is itself connected by law to the regularity. But not only does it seem absurd to think of the G-law as a cause, this would require a higher-level law to connect it to the generalization. A G law may G-explain instances of its associated regularity, but this explanation is nothing like usual scientific explanation.

If a $\mathrm{G}$ law doesn't cause instances of its associated regularity how is it related to them? As I mentioned earlier Emery suggests that a G-law metaphysically explains instances of its associated generalization by grounding them. Of course, a Humean cannot accept this since on the Humean account the HM grounds the laws so if the laws ground instances of the HM we are saddled with the circularity in explanation 
although now a circularity in metaphysical rather than scientific explanation. But on the governing view there is no circularity.

This is an intriguing proposal. If it can be developed and defended, then we would have an account of how a G-law is related to and explains its associated regularity. However, the relationship between a law and its instances doesn't seem like other cases of grounding and calling it so sheds no light on how it explains its instances. Five principles that are generally held regarding grounding is that if $A$ grounds $B$ then $A$ is more fundamental than $B, A$ necessitates $B, A$ explains $B$, grounding is transitive and nothing grounds itself. Most proponents of grounding (Bernstein (2018) calls them "groundhogs") say that the instances of a generalization ground the generalization. So the view is that G-laws ground their instances and that the instances ground generalizations.

I have a few worries about Emery's proposal. One is that probabilistic laws don't necessitate their instances so if necessitation of grounded by ground is required it fails. One wouldn't want to hold that non probabilistic laws ground their instances while probabilistic laws don't. Neither probabilistic not non probabilistic laws seem to be more fundamental than their instances in the way, say, that the fact that an object is red is more fundamental than that it is colored or the atoms that compose an object are more fundamental than the object they compose. Laws do explain their instances but not in the way that other instances in which the ground explains what it grounds. For example, an instance of a realizer of a functional property grounds an instance of the functional property in virtue of satisfying its functional role. But this model is not at all applicable to the relation between a law and its instances. For these reasons I am skeptical that identifying the relation between a law and its instances with grounding illuminates the relation of governance. In fact, calling this relation grounding seems to amount only to saying that it is an explanatory relation that is not causation but says nothing about how or why it is an explanatory relation. One might as well take the governing relation as a primitive.

That is exactly what Tim Maudlin does. He writes "My analysis of laws is no analysis at all. Rather, I suggest we accept laws as fundamental entities in our ontology. Or, speaking at the conceptual level, the concept of a law cannot be reduced to other more primitive notions". (Maudlin 2007, p.18). But if laws are claimed to be primitive entities and it is simply posited that a law guarantees the truth of an associated generalization then one rightly wonders whether such primitive laws provide any kind of explanation at all. Descartes accounted for the explanatory relation by attributing it to divine action. But if we want to do without God then I suggest we also should do without G-laws and make do with L-laws. 


\section{References}

Armstrong, D. 1983. What Is a Law of Nature? Cambridge: Cambridge University Press.

Bernstein, S. 2016. Grounding is not Causation. Philosophical Perspectives 30(1): 21-38.

Boghal, H. forthcoming. Nomothetic Explanation and Humeanism about Laws of Nature. Oxford Studies in Metaphysics.

Cohen, J.; Callender, C. 2009. A better best system account of lawhood. Philosophical Studies 145(1): 1-34.

Cartwright, N. 1999. The dappled world: a study of the boundaries of science. Cambridge: Cambridge University Press.

Cartwright, N. 2005. No God, No Laws. In: E. Sindoni; S. Moriggi (eds.) Dio, La Natura, e La Legge, pp.183-190. Milan: Angelicum.

Elga, A. 2000. Self-locating belief and the Sleeping Beauty problem. Analysis 60(2): 143-147.

Emery, N. 2019. Laws and their instances. Philosophical Studies 176(6): 1535-1561.

Foster, J. 2004. The Divine Lawmaker. Oxford University Press.

Harrison, P. 2019. Laws of God or Laws of Nature? Natural Order in the Early Modern Period. In: P. Harrison; J. Roberts (eds.) Science Without God?, pp.58-76. Oxford: Oxford University Press.

Hicks, M. T.; van Elswyk, P. 2015. Humean laws and circular explanation. Philosophical Studies 172(2): 433-443.

Hoefer, C. 2019. Chance in the World: A Humean Guide to Objective Chance. Oxford: Oxford University Press.

Jolley N. 2002. Occasionalism and efficacious laws in Malebranche. Midwest studies in philosophy 26(1): 245-257.

Lange, M. 2013. Grounding, scientific explanation, and Humean laws. Philosophical Studies 164(1): 255-261.

Lewis, D. 1986 [1980]. A Subjectivist's Guide to Objective Chance. In: Philosophical Papers, vol. 2, pp.83-132. Oxford: Oxford University Press.

Lewis, D. 1994. Humean supervenience debugged. Mind 103(412): 473-491.

Loewer, B. 1996. Humean Supervenience. Philosophical Topics 24: 101-127.

Loewer, B. 2000. Laws and Induction. Unpublished manuscript.

Loewer, B. 2001. Determinism and Chance. Studies in History and Philosophy of Science Part B: Studies in History and Philosophy of Modern Physics 32(4): 609-620.

Loewer, B. 2004. David Lewis's Humean Theory of Objective Chance. Philosophy of Science 71(5): 1115-1125.

Loewer, B. 2007. Laws and natural properties. Philosophical Topics 35(1/2): 313-328.

Loewer, B. 2012. Two accounts of laws and time. Philosophical Studies 160(1): 115-137.

Maudlin, T. 2007. The Metaphysics within Physics. Oxford: Oxford University Press.

Miller, E. 2015. Humean scientific explanation. Philosophical Studies 172(5): 1311-1332.

Ott, W.; Patton, L. 2018. Laws of Nature. Oxford: Oxford University Press.

Ott, W. 2019. Berkeley's Best System: An Alternative Approach to Laws of Nature. Journal of Modern Philosophy 1(1): 4.

Pettigrew, R. 2016. Accuracy and the Laws of Credence. Oxford: Oxford University Press.

Stanley, M. 2014. Huxley's church and Maxwell's demon: from theistic science to naturalistic science. Chicago: University of Chicago Press. 
Van Fraassen, B. C. 1980. The scientific image. Oxford: Oxford University Press.

Weinberg, S. 1993. Dreams of a Final Theory: The Search for the Fundamental Laws of Nature. London: Hutchinson Radius.

Weinberg, S. 1996. Sokal's Hoax. The New York Review of Books, Volume XLIII, No. 13, pp 11-15, August 8.

\section{Notes}

${ }^{1}$ The history of the development concept of law of nature is a fascinating and complicated story. An excellent discussion is Peter Harrison's "Laws of God or Laws of Nature". Descartes and Malebranche were advocates of the view (occasionalism) according to which God is the cause of all motion and change in motion of material bodies and laws describe God's action. Somehow laws became conceived of as themselves the causes of motion of matter as supervised by God. Malebranche suggests that laws are aspects of God's will that cause motion. See Jolley (2002). See also Ott and Patton (2018) collection on the history of the concept of laws of nature and Walter Ott's discussion of Berkeley on laws (2019).

${ }^{2}$ The simplicity and comprehensiveness of the system of laws was thought to reflect God's nature. Descartes even claimed to derive conservation laws a priori from God's nature.

${ }^{3}$ The theological origin of the concept of laws has mostly been forgotten by metaphysicians of science but not of course by historians of science. The story of the secularization of science and in particular the concept of law is complicated and fascinating. See Stanley (2014) for parts of this story.

4"Humean" because Hume was the philosopher who first clearly rejected the governing account. There is a third alternative that rejects laws as the engine of scientific explanation for powers and dispositions. On this approach systems possess powers and capacity to act in various ways and lawful generalizations emerge as generalizations of their interactions. See Cartwright (1999). This approach can be seen as a reversion to an Aristotelian conception of science that Descartes replaced with the view of a law governed universe.

${ }^{5}$ Two who do make the connection between theology and governing explicit but for different reasons are John Foster and Nancy Cartwright. Foster in The Divine Law Maker (2004) argues that God's will is required to make sense of the governing role of laws and Cartwright in "No God No Laws" (2005) appeals the connection between laws and theology in her argument that there are no laws of nature and for a return to a more Aristotelian account of science.

${ }^{6}$ Among recent proponents of the governing view are David Armstrong, Michael Tooley and Tim Maudlin. Recent proponents of the Humean view are David Lewis, John Earman and Barry Loewer.

${ }^{7}$ See Loewer (1996) for a discussion of Humean Supervenience. There are best systems accounts that reject the view that laws govern but differ from Lewis' account. See for example Loewer (2007) and Callender and Cohen (2009).

${ }^{8}$ In fact, Lewis' specific accounts are not successful. However, there are Humean accounts in similar spirit which look promising (see Loewer 2007).

${ }^{9}$ Lewis mentions simplicity and informativeness as features to be optimized. These are important but it is better to understand the account in terms of balancing the features that 
reflect practice in the scientific community. The criteria for what make a system optimal are best understood as being derived from the practice of fundamental physics.

${ }^{10}$ Lewis proposes measuring the informativeness of a probabilistic law in terms of how well the actual history "fits" it which is equated with P(actual history/Law). This account doesn't work (Cf. Elga 2000) but there are promising accounts of the informativeness of probabilistic claims in terms of how accuracy (e.g. Pettigrew 2016).

${ }^{11}$ Ott (2019) discusses how Berkeley's account of laws prefigures' Lewis' BSA.

${ }^{12}$ Lewis (1986) [1980] shows how to extend the account to probabilistic laws. His approach is modified and extended in Loewer (2004) and Hoefer (2019).

${ }^{13}$ Armstrong, Maudlin, Emery, Lange.

${ }^{14}$ Harjit Bhogal in "Nomothetic Explanation and Humeanism about Laws of Nature" (forthcoming) develops the distinction between scientific (he calls "nomothetic") explanation into a general defense of Humean accounts of laws against the numerous objections in addition to the explanatory circle objection that anti-Humeans have pressed against Humean accounts.

${ }^{15}$ Lange's example of fitness.

${ }^{16}$ Mike Hicks and Peter van Elswick in "Humean Laws and Circular Explanation" (2015) and Elizabeth Miller in "Humean Scientific Explanation" (2015) extensively discuss Loewer's response to the circularity objection. They provide counterexamples to Trans and show that it doesn't apply to L-laws.

${ }^{17}$ There are well known problems with spelling out the DN account so as to make it immune to counter examples. (van Frassen 1980). But whether or not this can be done successfully it is certainly the case that some D-N arguments are explanations.

${ }^{18}$ Governing in its ordinary uses is a causal notion. A sovereign governs her subjects by issuing edicts that are involved in causing behavior. But G-laws don't govern like that. On nomological accounts of causation it would require higher level laws to connect a G-law with its instances.

${ }^{19}$ The argument that follows is based on John Foster's discussion in his The Divine Lawmaker. Foster further argues from that an anti-Humean account of laws (and a Deity to enforce the laws) is required to demonstrate the rationality of inductive inference. I respond to this line of thought in "Laws and Induction" (2000).

${ }^{20}$ Actually, there are just as many systematizable worlds as unsystematizable worlds. Both sets are uncountable. So, the POI requires a measure for its application.

\section{Acknowledgments}

Thanks to Mike Hicks, Peter Harrison, Heather Demarest, Nina Emery, and Jonathan Schaffer (who saved me from a couple of errors). 